\title{
Enseigner la grammaire en Lozère, retard de l'alphabétisation et retard pédagogique
}

Pierre Boutan

\section{(2) OpenEdition}

1 Journals

Édition électronique

URL : http://journals.openedition.org/trema/1873

DOI : 10.4000/trema.1873

ISSN : 2107-0997

Éditeur

Faculté d'Éducation de l'université de Montpellier

\section{Édition imprimée}

Date de publication : 1 décembre 1997

Pagination : 117-124

ISSN : 1167-315X

\section{Référence électronique}

Pierre Boutan, « Enseigner la grammaire en Lozère, retard de l'alphabétisation et retard pédagogique », Tréma [En ligne], 12-13 | 1997, mis en ligne le 24 novembre 2010, consulté le 19 avril 2019. URL: http://journals.openedition.org/trema/1873 ; DOI : 10.4000/trema.1873

Ce document a été généré automatiquement le 19 avril 2019

Trema 


\title{
Enseigner la grammaire en Lozère, retard de l'alphabétisation et retard pédagogique
}

\author{
Pierre Boutan
}

1 La mise en place, surtout avec la Monarchie de Juillet, d'une institution d'État pour contrôler l'Instruction publique, visait, comme le dit Guizot lui-même, à "gouverner les esprits ",non seulement ceux de la future classe dirigeante, mais aussi ceux du plus grand nombre, les enfants du peuple des villes et des campagnes. Cela passe bien entendu, par la recherche d'une fréquentation généralisée des écoles, et aussi la volonté renforcée d'intervenir sur les contenus et les formes de l'enseignement. Le ministère de l'Instruction publique se donne plusieurs moyens d'intervention. Il organise la formation des maîtres : examen du brevet de capacité nécessaire pour enseigner, formation initiale par les écoles normales d'instituteurs, mais en délaissant davantage la formation du personnel féminin. Formation continue aussi, en particulier par la généralisation de conférences pédagogiques, qui s'éteignent cependant progressivement dans les années 1840. Il instaure un corps nouveau d'inspecteurs primaires, développe un organe périodique à destination de tous les maîtres du territoire, chargé de faire connaître ses vues en matière de contenus et de pratique pédagogique. Il s'efforce enfin de contrôler, avec un succès limité, les « livres classiques », c'est-à-dire les manuels scolaires.

Compte tenu de son importance économique et politique, l'enseignement de la langue nationale est au premier rang des objectifs ministériels. Mais le caractère minoritaire de son usage populaire sur le territoire national pose un sérieux problème, surtout bien sûr dans les campagnes. Il peut sans doute expliquer qu'il faille attendre le modèle national de règlement des écoles publiques, consécutif à la loi du 15 mars 1850 (dite loi Falloux), pour voir apparaître un article prescrivant officiellement partout l'usage du seul français dans les écoles. 


\section{Éléments de description de la ruralité pédagogique}

Remarque initiale : il faut constater que la direction de l'Instruction publique va résister à toutes les pressions, et parfois au plus haut niveau, pour faire des programmes différenciés entre villes et campagnes ${ }^{1}$. Du coup, le modèle urbain n'en est que plus prestigieux, et, de fait, c'est de la ville que viennent les grandes innovations depuis au moins le XVII siècle et les frères des écoles chrétiennes de Jean-Baptiste de la Salle.

4 On soulignera donc les conditions favorables à la ville, en particulier avant les débuts de la III République :

- les moyens financiers, permettant l'équipement des écoles, le recrutement des meilleurs maîtres ;

- l'importance des effectifs d'élèves, qui peuvent favoriser les possibilités de constitution de divisions homogènes, à l'origine des écoles à plusieurs maîtres et plusieurs classes, rares même en milieu urbain encore vers 1850-1860 ;

- l'environnement linguistique, beaucoup plus facilitant pour enseigner la langue nationale ;

- la proximité des lieux d'échanges culturels, ce qui, dans le domaine pédagogique, concerne plus particulièrement les librairies où arrivent les nouveaux manuels. La situation évolue après la guerre de 1870 : ainsi Armand Colin fait sensation en adressant la fameuse Grammaire de Larive et Fleury à tous les instituteurs de France (1871), inaugurant ainsi la pratique de l'envoi de spécimens gratuits.

\section{La situation de la Lozère}

La Lozère fait partie de ces départements géographiquement défavorisés (éloignement des grandes voies naturelles de communication, absence de villes importantes...), en situation nettement allophone par rapport à la langue nationale. Au sud de la fameuse ligne Saint-Malo-Genève (dite aussi ligne Maggiolo), elle fait souvent figure d'exemple en matière de retard dans tous les domaines. En 1879, Paul Bert cite à la tribune de l'Assemblée nationale le rapport de 1864 de l'inspecteur d'académie de la Lozère : « Notre département est le plus ignorant et le plus arriéré de toute la France " ${ }^{2}$. Jugement excessif sans doute, alors même que la Lozère est à la même date au $9^{\mathrm{e}}$ rang pour le nombre des inscrits par rapport à la population scolarisable... Mais on sait que la régularité de la fréquentation est une donnée bien différente du nombre des inscrits, et il faut aussi tenir compte qu'alors la fiabilité des statistiques est fortement sujette à caution.

Quel rapport entre retard dans l'alphabétisation et retard dans les contenus et les formes de l'enseignement? On trouve ici un débat classique entre hiérarchie ${ }^{3}$ et instituteurs. La hiérarchie reconnaît sans doute comme cause à l'insuffisance de la fréquentation scolaire le manque d'effort des familles et des élus locaux pour améliorer les équipements scolaires, les rétributions et les conditions de travail des maîtres.

7 Mais elle met aussi en avant, en s'appuyant d'ailleurs sur des exemples patents, que la qualité de l'enseignement, et du maître, est un facteur d'importance pour favoriser la fréquentation. On devine bien entendu que les maitres, de leur côté, ne l'entendent généralement pas ainsi, ce qui se traduit très souvent par la passivité, voire l'hostilité à peine dissimulée, face aux mesures d'organisation des études commandées par la hiérarchie. Ainsi la routine, pour parler le langage de l'époque, est indéniablement en 
rapport avec les modalités de fréquentation, et plus généralement avec la pression sociale variable avec laquelle les parents investissent le domaine scolaire.

Pour témoigner de la situation réelle, on dispose entre autres des rapports d'inspection, rapports qualitatifs mais aussi quantitatifs, quand il s'agit de recenser les manuels présents dans les classes, souci fréquent des autorités depuis au moins l'enquête Guizot de 1833. On s'en tiendra ici à l'occasion qu'offre un relevé de titres d'ouvrages effectué en 1858 dans l'arrondissement de Florac, conservé aux archives départementales de la Lozère (1 T 220).

Rappelons au passage qu'il s'agit de la partie du département la plus avancée du point de vue de l'alphabétisation, ce qui n'est sûrement pas sans rapport avec la concurrence religieuse entre catholiques et protestants, absente ailleurs ${ }^{4}$.

\section{L'enseignement de la grammaire}

D'autre part, on s'intéressera essentiellement aux manuels de grammaire, correspondant à un enseignement qui fait alors partie des hautes études de l'école primaire. Dans le cursus décrit par la loi Guizot et les programmes d'études qui en résultent, la grammaire fait partie des "Éléments de la langue française », et renvoie à la seule première division, la dernière de l'école primaire 5 .

11 Ainsi, une fois qu'on a appris à lire puis à écrire, on apprend orthographe et grammaire, le sommet des études étant constitué par les « exercices de style ", soit, comme on dirait aujourd'hui, la maitrise de certaines productions écrites. Bien entendu, le cursus complet n'est parcouru que par une très faible minorité des élèves, moins encore en Lozère comme on va le voir.

Insistons sur un autre point: le livre de grammaire est indispensable à l'élève, compte tenu des formes habituelles de son enseignement. Il faut en effet considérer comme sans effet la circulaire Rouland de 1857, qui invitait à une démarche intuitive, à l'opposé des pratiques courantes, en affirmant souhaiter que l'on ne mette " point de grammaire entre les mains des élèves». Laréitération périodique par d'autres textes officiels des mêmes principes pendant les trente années qui suivent, assortie du constat désolé par les autorités de son défaut d'application, est un signe clair de l'inefficacité d'une circulaire qui fut plus fameuse pour les inspecteurs que pour les maitres.

13 La leçon de grammaire ne consiste qu'à réciter par cœur une ou deux pages du livre devant le maître ${ }^{6}$. Encore en 1876, l'inspecteur d'académie de la Lozère décrit "le système vicieux qui préside à cet enseignement. On récite en effet sa leçon de grammaire comme un texte d'auteur quelconque, ou comme sa leçon de catéchisme [...]. Quant aux explications orales, à des exercices sur le tableau noir, à des séries d'exemples où l'élève tire lui-même la règle, il n'en est pas question; non plus que des dictées méthodiques sur les sciences usuelles, ou des narrations simples à la portée des enfants ou de petites lettres à leurs amis, à leurs parents, etc. " ${ }^{7}$

14 Les manuels de grammaire sont d'ailleurs parfois présentés sous une forme destinée à faciliter l'organisation de cette récitation, en utilisant l'apparence d'un dialogue où le maître pose les question et l'élève répond par la récitation mot à mot du paragraphe adéquat. Ainsi la Grammaire des Frères des écoles chrétiennes contient une mise en forme sur un modèle catéchistique transparent ( $C f$. doc. $\left.\mathrm{N}^{\circ} \mathbf{1}\right)$. 

relation de la leçon (toujours au sens que nous venons d'évoquer) avec l'orthographe par les livres d'exercices correspondants, elle postule, selon le modèle qui connaît un immense succès avec la Grammaire de Noël et Chapsal (1 ${ }^{\text {re }}$ édition en 1823), l'emploi et donc l'achat, d'un manuel spécifique. Même si commencent à exister des ouvrages associant leçon et exercices, tel la Lexicologie des écoles, qui fait d'abord connaître Pierre Larousse, à partir de 1856.

\section{Document 1.}

Pages 1 et 3 de l'ouvrage cité en référence ci-dessous. (Collection particulière) ; reproduction de ces pages, grâce à l'aimable autorisation du collectionneur.

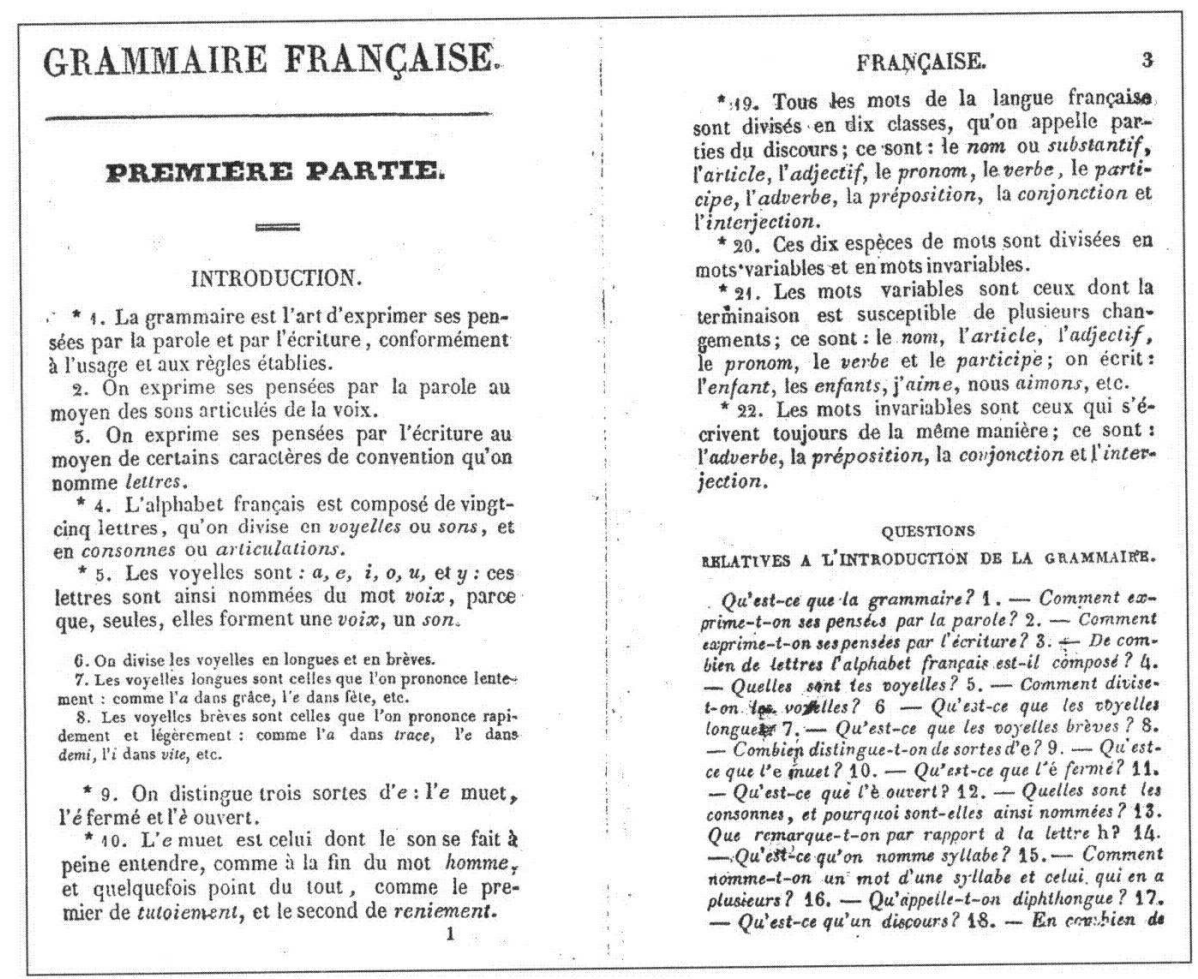

F.P.B., Grammaire française élémentaire, suivie d'une méthode d'analyse grammaticale raisonnée, à I'usage des écoles chrétiennes, Mame (Tours) et Poussièlgue-Rusand (Paris), Vingtième édition, 1843, $212 \mathrm{p}$.

\section{Les différences entre Nord et Sud}

Venons-en à l'examen de la répartition des titres de notre liste de 1858 (Cf. doc. $\mathbf{N}$ 2). On la mettra en valeur en la comparant avec une enquête sur le même objet, conduite dans l'Oise en 1852 en s'en tenant à l'arrondissement de Senlis, dans des conditions statistiques non identiques, mais qui excluent cependant des différences insurmontables ${ }^{8}$. Dans l'Oise 
donc, 178 instituteurs et institutrices remplissent un questionnaire détaillé : tous font état de la présence d'un titre de livre de grammaire, et parfois de deux.

Document 2.

Titres des ouvrages de grammaire utilisés dans la circonscription de Florac (Lozère) en 1858. Tableau tiré de la liste... signée par l'inspecteur Maurin le 11 juillet 1858.

Source : Archives Départementales de la Lozère, 1 T 220.

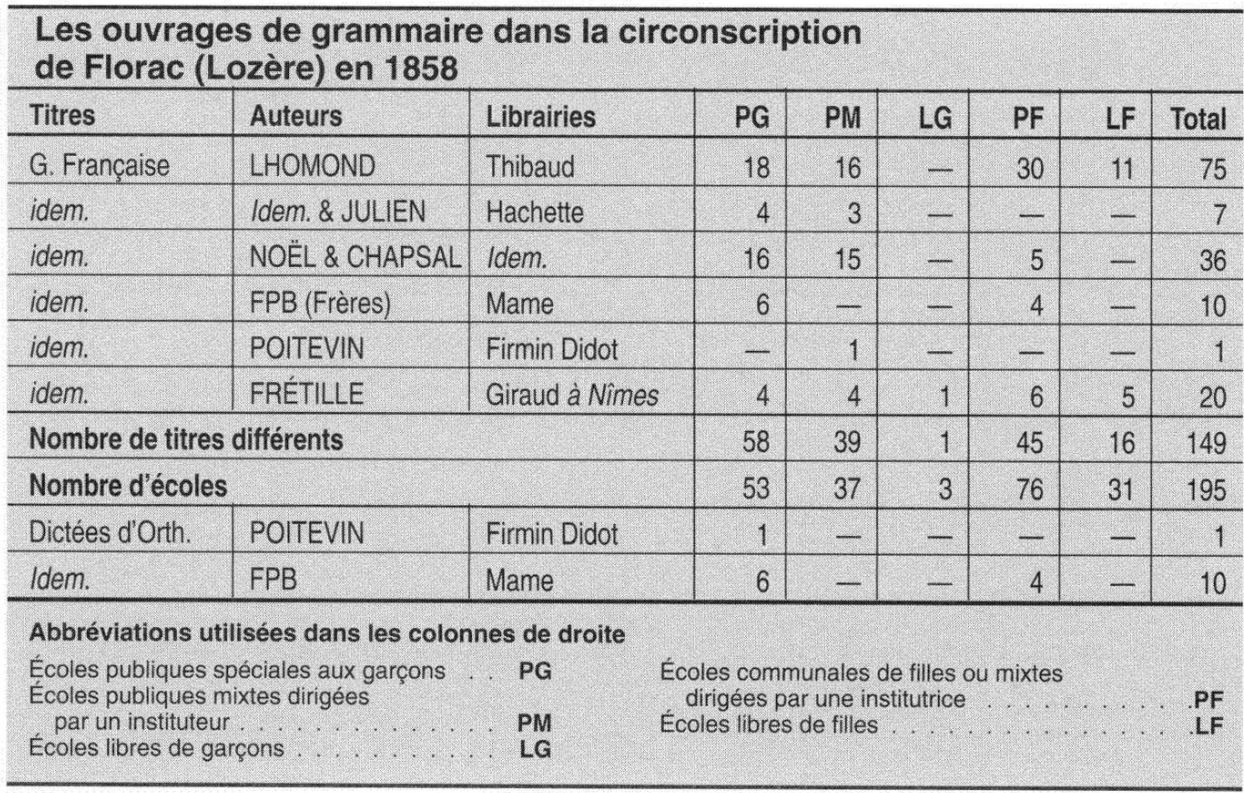
recensées, mais on ne trouve que 149 titres d'ouvrages de grammaire. C'est dire qu'au moins 46 écoles, soit près du quart, n'ont pas la possibilité d'un enseignement de cette nature. 
elles ne sont pas nécessairement significatives, dans la mesure où il s'agit le plus souvent d'adaptations du célèbre ouvrage d'un des plus grands auteurs pédagogiques, dont la première édition date de $1780^{\circ}$.

Le retour à Lhomond est typique de la fin des années 1840 et s'impose officiellement dans les écoles normales après la loi Falloux. Il est jugé en effet beaucoup plus simple - mais aussi moins ambitieux sinon pauvre - que le Noël et Chapsal qui connaît un succès commercial considérable depuis 1823.

Si l'on mesure donc la place de Noël et Chapsal en nombre de titres recensés, on trouve un pourcentage semblable à Senlis comme à Florac, soit environ un quart. Mais la répartition n'en est pas identique. Dans l'Oise ce sont les écoles situées dans les plus petites communes qui sont les plus grandes utilisatrices, par comparaison avec celles des communes les plus peuplées. Et si l'on compare l'équipement des écoles selon le sexe, alors on voit que les écoles de filles ont plus souvent Noël et Chapsal que Lhomond. Sachant que les écoles des petites communes d'une part, les écoles de filles d'autre part, sont considérées comme les moins avancées dans la qualité de leur enseignement, du fait concomitant de leur pauvreté commune, on a là un indice cohérent. Au contraire, en Lozère, les écoles de l'arrondissement de Florac n'en sont pas encore à voir refluer l'usage de l'ouvrage de Noël et Chapsal dans l'enseignement de la grammaire. Et on ne sera pas surpris d'apprendre qu'en 1876 encore, l'inspecteur d'académie de la Lozère, dans le même rapport annuel déjà cité, déplore que l'on en soit toujours à cet ouvrage dans son département.

On peut donc en conclure des différences très nettes entre deux arrondissements situés au Nord et au Sud, différences à leur tour ralenties ou accentuées selon que l'on a affaire aux écoles de filles ou de garçons. Au Nord, il est difficile de distinguer quantitativement écoles de filles, écoles de garçons, écoles mixtes. Au Sud, les institutrices sont nettement en retard par rapport aux instituteurs. La présence de livres d'exercices qui, dans l'Oise, discriminent les maîtres faibles dans les petites communes pauvres, est ici à l'inverse : seules sans doute les écoles des communes les plus importantes, où d'ailleurs se rapprochent filles et garçons, en sont pourvues.

La situation est confirmée quelque vingt ans plus tard encore de cette différence entre filles et garçons. Le Bulletin spécial et officiel de l'instruction publique pour la Lozère, publiant en 1876 un tableau de l'emploi du temps dans les écoles primaires, indique en Nota : "Dans les écoles de filles, il y aura dictée à l'heure consacrée à la grammaire française »"

Ainsi voit-on apparaître, au-delà des pôles ville - campagne, d'autres différenciations que la fin du siècle s'emploiera à combler : Oppositions Nord et Est-Sud et Ouest, filles-garçons (qu'on peut alors confondre avec instituteurs-institutrices), et, ce qui n'est pas alors bien visible en Lozère au milieu du siècle, laïques-congréganistes. Il n'est pas sans intérêt de souligner que les différences quantitatives et qualitatives en matière d'enseignement tendent à se recouper, même si ce n'est pas de manière mécanique. D'où l'hypothèse sous-jacente : la « routine » ne serait-elle pas le mode d'adaptation trouvé par les maîtres à des conditions spécialement difficiles d'enseignement?

Il y a en tout cas tout lieu de penser que la ruralité pédagogique est bien un élément de plus à ajouter, encore au milieu du XIX siècle, au constat de l'infériorité des campagnes par rapport au progrès économique et social du pays. 


\section{BIBLIOGRAPHIE}

BOUTAN P., in « Histoire de l'enseignement du français à l'école primaire élémentaire de 1850 à 1900 », thèse d'État, Paris V, 1994, 2 v., 798 p. ; consultable au Centre de documentation en histoire de l'éducation de l'IUFM de Montpellier, et aux Archives départementales de la Lozère.

Pour une présentation plus succincte, voir BOUTAN P., La langue des Messieurs. Histoire de l'enseignement du français à l'école primaire, Armand Colin, collection « Formation des enseignants » 1996. $256 \mathrm{p}$.

\section{NOTES}

1. Si l'on excepte l'enseignement de l'agriculture, qui sera spécifique aux écoles rurales avec une épreuve au Certificat d'études, au début du XX $\mathrm{X}^{\mathrm{e}}$ siècle.

2. Cité dans La semaine religieuse de Mendedu 27 février 1880, p. 136, dans un article intitulé «Ce que pensent de la Lozère M.P. BERT, un inspecteur d'Académie d'autrefois et le Moniteur d'aujourd'hui ».

3. Précision de vocabulaire : la hiérarchie est le terme qui servira à désigner globalement par commodité les membres de l'inspection, soit pour l'essentiel les inspecteurs primaires, les plus nombreux, en principe un par arrondissement.

4. Voir le mémoire de PETRE C., déposé aux archives de la Lozère.

5. Qui en comporte trois, soit autant que les fameux « cycles » de la loi actuellement en vigueur (1989)...

6. D'où l'intérêt, pas seulement économique, d'avoir des livres courts (les Élémens de grammaire françaisede LHOMOND, manuel assurément le plus répandu au début du siècle, a 80 pages).

7. «Rapport sur la situation de l'enseignement primaire 1876 », in Archives Départementales de la Lozère, $1 \mathrm{~T} 56$.

8. A.D. de l'Oise, 1 T 3115 à 3117.

9. Rappelons que les Élémens de grammaire françaisede LHOMOND avaient étés retenus comme grammaire de référence au terme des concours d'ouvrages classiques de l'époque révolutionnaire. La longévité des ouvrages de Lhomond en matière d'enseignement du latin est encore plus grande, puisque son De virisétait encore récemment réédité.

10. $\mathrm{N}^{\circ} 3$ du 15 mai 1876, p. 67. Il s'agit, avec les travaux d'aiguille (pris cette fois sur l'horaire de grammaire) de la seule différence selon les sexes. C'est la preuve que l'enseignement de l'orthographe peut disposer, même s'il s'agit d'un cursus archaïque, de son autonomie propre.

\section{RÉSUMÉS}

Non disponible 
Not available

INDEX

Mots-clés : alphabétisation, école primaire, grammaire, IIIe république, Lozère, ruralité

Keywords : grammar book, grammar method, primary school, rural context, rural life, third republic, wiping out illiteracy

\section{AUTEUR}

\section{PIERRE BOUTAN}

IUFM de Montpellier 